Quaderni
QUADEDENII $\begin{aligned} & \text { Communication, technologies, pouvoir } \\ & \text { Colères politiques }\end{aligned}$

\title{
Colères politiques
}

\section{Thierry Devars}

\section{(2) OpenEdition \\ Journals}

Édition électronique

URL : https://journals.openedition.org/quaderni/2102

DOI : 10.4000/quaderni.2102

ISSN : 2105-2956

\section{Éditeur}

Les éditions de la Maison des sciences de l'Homme

Édition imprimée

Date de publication : 15 octobre 2021

Pagination : 9-16

Référence électronique

Thierry Devars, « Colères politiques », Quaderni [En ligne], 104 | Automne 2021, mis en ligne le 15 octobre 2021, consulté le 08 novembre 2021. URL : http://journals.openedition.org/quaderni/2102 DOI : https://doi.org/10.4000/quaderni.2102 


\section{Avant-propos: Colères politiques}

Thierry Devars

CELSA - Sorbonne Université GRIPIC 
Omniprésente dans le champ politico-médiatique, la colère constitue une force vive du débat public contemporain. Individuelle ou collective, elle incarne la puissance vitale des émotions humaines, entendues au sens étymologique du terme "motio", signifiant à la fois le "mouvement" et la "fièvre". C'est cette dynamique phénoménologique, intimement liée au corps et à la sensorialité, qui la rend perceptible et lisible. Considérant le large spectre des motivations qui la sous-tendent, la colère échappe à l'horizon restrictif d'un désir irraisonné de nuisance, ou pour reprendre les mots de Spinoza, à "l'effort de causer du mal à l'objet de notre haine'." A contrario, la colère peut être aiguillée par une raison d'être susceptible d'asseoir sa légitimité. Dans le cadre d'un conflit interpersonnel, elle peut ou non donner raison à celle ou celui qui l'exprime et contribuer à réguler les rapports de pouvoir entre ses parties prenantes. Dans une perspective sociale plus globale, la colère interroge et met à l'épreuve les principes et valeurs structurants du collectif. Elle peut ainsi exprimer la défense de certains idéaux, au premier rang desquels figure l'idéal démocratique de justice, et revêt en ce sens une dimension fondamentalement politique.

La question de l'acceptabilité sociale de la colère, tour à tour émotion aveugle et passion raisonnable, s'inscrit à la croisée de deux dynamiques a priori divergentes: d'une part, en ce qu'elles contreviennent potentiellement à la stabilité de l'ordre social et à la préservation d'un idéal de civilité, ses formes expressives les plus manifestes suscitent méfiance et réprobation, d'autre part, c'est paradoxalement au nom même de la défense du collectif qu'elle entend revendiquer sa légitimité et sa raison d'être. Émotion ambivalente, la colère constitue ainsi une forme expressive à part entière du/de la politique, entendu dans sa dimension normative (le politique recouvre le "lieu des valeurs symboliques" ", l'espace "de résolution des conflits et d'arbitrage des intérêts divergents de la société ${ }^{3}$ ") et sa dimension agonistique (la politique renvoie à "la lutte concurrentielle pour la répartition du pouvoir" ").

\section{LA FABRIQUE MÉDIATIQUE DES COLÈRES POLITIQUES}

Inscrit dans le cadre d'une réflexion interdisciplinaire portant sur le caractère politique des émotions, ce numéro de Quaderni se propose de saisir la colère à l'intérieur et au-delà du champ politique stricto sensu. II entend appréhender l'unité et la cohérence d'une émotion à la fois spectaculaire et fugace, reconnaissable et diffuse, individuelle et/ou collective, à travers l'éclectisme de ses manifestations sensibles. Dans le sillage d'une approche construc-

1. Baruch Spinoza, L’Éthique, Traduction par Charles Appuhn, Paris, Garnier Frères, (1677) 1913, p.334.

2. Patrick Charaudeau, Le Discours politique. Les masques du pouvoir, Paris, Vuibert, 2005, p. 236.

3. Rémi Lefebvre, Leçons d'introduction à la science politique, Paris, Ellipses, 2010, p. 13.

4. Ibid., p.14. 
tiviste attentive à la fabrique sociale des émotions, le numéro se propose d'interroger plus spécifiquement le rôle joué par les médias d'information classiques et numériques qui, en déterminant la morphologie de la sphère publique contemporaine, infléchissent également les conditions de mise en visibilité des colères politiques. Entendue au sens large du terme, la scène politique contemporaine ${ }^{5}$ constitue un espace privilégié d'observation des manifestations de la colère, tant à l'échelle individuelle de la communication des professionnels de la vie politique qu'à celle des mobilisations collectives et dynamiques d'opinions qui contribuent pleinement à la "politisation" des questions d'intérêt général.

Le spectre des objets susceptibles d'intégrer une approche interdisciplinaire du caractère politique de la colère est vaste: des colères codifiées et ritualisées des professionnels de la vie politique (pensons aux "colères saines" de Ségolène Royal ou aux tweets rageurs de Donald Trump) aux mouvements collectifs contestataires (parmi les plus récents, les Gilets Jaunes, \#MeToo ou Black Lives Matter) en passant par certaines virulentes dynamiques d'opinions exprimées sur les réseaux socio-numériques (à l'image des discours complotistes de QAnon et des florissantes revendications "antisystème "). La mosaïque de ces formes expressives, des contextes situés dans lesquels survient la colère et des processus d'assignation et de lecture qui accompagnent ses manifestations publiques nous autorise à penser ensemble son caractère irréductiblement singulier (la colère) et la pluralité des modes de construction sociale dont elle peut faire l'objet dans le cadre d'une sphère publique dominée par les logiques médiatiques (les colères).

Suivant l'approche proposée par Christian Le Bart dans son ouvrage sur les émotions politiques, la colère peut être appréhendée "à partir de ses manifestations corporelles les plus indiscutables ", en d'autres termes à partir de ses formes expressives et des contextes qui leur permettent d'advenir. Méthodologiquement, les traces de la colère constituent un fil d'Ariane susceptible d'une part de fédérer au sein d'un même numéro une série de contributions d'inspirations disciplinaires diverses, et d'autre part de penser son caractère politique à l'intérieur et au-delà du champ d'acteurs auquel elle peut spontanément être associée.

\section{COLÈRES DES POLITIQUES: UNE ÉMOTION SOUS CONTRAINTE}

Par-delà les enjeux propres à la saisie médiatique des émotions, ce numéro propose une réflexion sur les colères exprimées individuellement par les professionnels de la politique. Partant des recherches en sciences humaines et sociales sur le rôle des émotions dans le champ politique, les contributions de Marion

5. Philippe Braud, L’Émotion en politique, Paris, Presses Universitaires de Sciences Po, 1996.

6. Christian Le Bart, Les Émotions du pouvoir. Larmes, rires, colères des politiques, Paris, Armand Colin, Paris, 2018, p. 24. 
Ballet et Thierry Devars ont pour objectif de questionner en son sein l'actualité des normes relatives aux colères exprimées par ses acteurs. L'édification au long cours du champ politique, corrélée à l'affirmation démocratique d'une sphère publique autonome, s'est accompagnée d'une codification tacite de formes expressives, admises ou proscrites, par et pour ses parties prenantes. Avec le déploiement généralisé d'une "société hyper-médiatisée7", l'arrière-plan de l'exemplarité de la parole publique s'articule désormais à des injonctions médiatiques susceptibles de revaloriser la colère.

C'est à la lumière de cette évolution au long cours des cadres de représentation des émotions exprimées par les politiques que Marion Ballet se propose d'analyser le rôle de la colère comme levier de mobilisation pour les candidats à l'élection présidentielle française entre 1981 et 2017. S'appuyant sur l'étude comparative d'un corpus de $\mathbf{4 5 9 5}$ messages électoraux émanant de documents de propagande constants, sa contribution montre que la colère obéit à trois fonctions distinctes. Elle incarne à la fois un outil de légitimation, un levier de réassurance militante et un mode d'identification partisane. Communément associée à l'expression des mécontentements populaires, la colère trouve progressivement sa place dans la communication des acteurs politiques au point de devenir un passage obligé des discours électoraux. Si la colère fait l'objet d'un processus de légitimation dans le champ politique, Marion Ballet montre qu'elle demeure toutefois peu compatible avec la fonction présidentielle et qu'elle constitue avant tout un marqueur idéologique privilégié des partis protestataires.

Dans la continuité de cette approche attentive à l'évolution des normes expressives de la colère, la contribution proposée par Thierry Devars met en lumière les injonctions paradoxales auxquelles doit faire face le chef de l'État dans l'expression publique de ses émotions. S'appuyant sur un corpus de 114 articles de presse centrés sur les premières années de mandature d'Emmanuel Macron, l'article interroge les modes de visibilité et la paradoxale discrétion des colères présidentielles dans l'espace public. Émotion globalement proscrite de la communication officielle, la colère est le plus souvent reléguée en coulisses. Si elle échappe aux logiques du spectacle médiatique, elle constitue toutefois un objet d'attention privilégié des discours journalistiques qui, par la médiation du off, confortent leur autorité testimoniale et définissent le régime de dicibilité des émotions exprimées par le chef de l’État. Rappelant les normes expressives auxquelles doit se conformer le président, ce travail médiatique de mise en présence des colères

7. Mario Carlòn, "Sur les pas de Verón... Une approche des nouvelles conditions de circulation du sens à l'ère contemporaine ", Traduction de Gustavo Gomez-Mejia \& Odile Cortinovis, Communication \& langages, $n^{\circ} 196$, Eliseo Verón. Vers une sémio-anthropologie, 2018. 
contribue en filigrane à la reconnaissance publique de l'autorité présidentielle.

LES RÉSEAUX DE LA COLÈRE : UNE ÉMOTION POLITIQUE

Par-delà le champ restrictif des émotions exprimées individuellement par les professionnels de la politique, la colère peut être plus largement saisie dans le cadre des mobilisations collectives et des mouvements d'opinions contemporains ${ }^{8}$. Substantif omniprésent dans les discours médiatiques pour qualifier les formes diverses de protestations et de controverses, la colère constitue en effet une puissance vitale, à l'intensité et à la morphologie variables, susceptible de fédérer des revendications collectives (pétitions, manifestations, grèves...). Les rapports de pouvoir dont elle témoigne entre celles et ceux qui détiennent et exercent l'autorité et celles et ceux qui la contestent ou la revendiquent lui assignent une fonction stratégique. Exprimée collectivement, la colère n'échappe pas à sa réputation sulfureuse. Sa capacité à mettre en péril d'une part la stabilité de l'ordre social et d'autre part la lisibilité et la légitimité de revendications collectives impose aux acteurs de la scène politique un travail appareillé de domestication.

Dans la continuité d'un ouvrage collectif de recherche conduit sur la pratique du débat d'actualité en ligne', la contribution d'Arnaud Mercier se propose d'analyser l'expression numérique des colères citoyennes sur des sujets d'actualité touchant à la vie politique, économique et sociale. Partant d'un vaste corpus de tweets diffusés ces dix dernières années, l'article entend appréhender à la fois les raisons et les modalités expressives des colères numériques, en considérant d'une part le contexte diffus de ressentiment susceptible de leur donner corps et d'autre part les contraintes et opportunités stylistiques offertes par le réseau social. Arnaud Mercier montre que la forte présence de la colère dans les débats d'actualité en ligne témoigne d'une profonde adéquation entre les caractéristiques expressives de Twitter, propice à l'épanchement des affects, et le climat de défiance à l'endroit du politique et des mutations socio-économiques contemporaines.

S'appuyant sur un terrain de recherche également consacré à l'expression de la colère en contexte numérique, la contribution de Lucie Raymond se propose d'analyser la rhétorique du ressentiment inscrite dans les revendications "antisystème" en ligne en se concentrant plus spécifiquement sur la figure du polémiste Alain Soral.

8. Christophe Traïni (dir.), Émotions... Mobilisation!, Paris, Presses de Sciences Po, 2009. Christophe Traïni et Loïc Blondiaux (dir.), La Démocratie des émotions. Dispositifs participatifs et gouvernabilité des affects, Paris, Presses de Sciences Po, 2018.

9. Arnaud Mercier, Nathalie Pignard-Cheynel (dir.), \#info. Commenter et partager l'actualité sur Twitter et Facebook, Paris, Éditions de la Maison des sciences de I'homme, 2018. 
Mise au service d'un discours revendiquant la rupture avec un certain ordre établi, la virulence de la colère se présente comme un instrument d'exclusion tout en consolidant l'identité des communautés rassemblées autour d'une même lecture du monde et d'un même motif de mécontentement. Inscrite en sciences de l'information et de la communication, la contribution de Lucie Raymond montre comment la "logique ressentimentiste ${ }^{10}$ " déployée dans le discours d'Alain Soral constitue le socle d'une idéologie à la fois inclusive et excluante, susceptible d'assigner une rationalité et une légitimité à un ensemble disparate de colères agrégées.

\section{MORPHOLOGIE DE LA COLÈRE : “MÉDIATIQUE ” D’UNE ÉMOTION}

\section{SPECTACULAIRE}

Considérant la colère à partir de ses traces distinctives, l'observation ethnographique in situ et l'approche sémiotique constituent des méthodes d'analyse susceptibles de dénaturaliser les discours et représentations dont elle fait communément l'objet dans la vie sociale. L'horizon d'une lecture critique des "combles d'artifices "1" à travers lesquels elle peut se donner à lire permet d'évacuer l'impasse d'une approche strictement symptomatique et physiocentrée de la colère. La construction sociale du caractère politique des émotions invite plutôt à considérer leurs manifestations extérieures en relation avec les contextes divers dans lesquels elles peuvent advenir. Le corps ne saurait donc constituer un espace de réclusion de la colère. Il se présente au contraire comme un support observable de médiation des rapports de pouvoir qui structurent le collectif et se rappelle à la dimension fondamentalement sociale des émotions humaines.

Dans cette perspective théorique, la contribution de Rym Gerwig-Kirèche et Philippe Marion se propose d'accorder une attention spécifique aux régimes scénographiques, narratologiques et médiatiques de mise en visibilité des corps dans l'expression des colères citoyennes. L'article s'appuie sur l'analyse comparative de deux mouvements politiques contestataires: d'une part, celui des Gilets Jaunes, d'autre part, celui des partisans de Donald Trump dans le contexte post-électoral du scrutin présidentiel de 2020. La proposition des auteurs $s^{\prime}$ inscrit dans la continuité de recherches conduites en narratologie des médias ${ }^{12}$ et en sciences de l'information et de la communication ${ }^{13}$. Elle se concentre plus spécifiquement sur l'analyse sémio-politique de deux débordements

10. Marc Angenot, "Nouvelles figures de la rhétorique: la logique du ressentiment", Questions de communication, $\mathrm{n}^{\circ} 12,2007, \mathrm{p} .67$.

11. Roland Barthes, Leçon, Paris, Seuil, (1977) 2015.

12. Philippe Marion, "Narratologie médiatique et médiagénie des récits", Recherches en communication, ${ }^{\circ} 7, \mathrm{UCL}$, Louvain-la-Neuve, 1997.

13. Rym Gerwig-Kirèche, De l'espace de la scène à l'espace de la page: médiation et médiatisation du corps de mode à travers le défilé et les séries photographiques de la presse magazine, Thèse de doctorat en Sciences de l'information et de la communication, Sorbonne Université, 2016. 
"colériques" abondamment commentés dans les médias: d'une part, la dégradation de l'Arc de Triomphe le $1^{\text {er }}$ décembre 2018 lors d'une manifestation des Gilets Jaunes, d'autre part, l'assaut lancé sur le Capitole par des partisans de Donald Trump le 6 janvier 2021. Attentif au potentiel narratogénique de ces colères collectives et spectaculaires, l'article entend montrer comment les performances corporelles des manifestants contribuent à la reconfiguration symbolique et politique des espaces investis. 


\section{BIBLIOGRAPHIE}

BACOT Paul, GABORIAUX Chloé, LE BART Christian et MAYAFFRE Damon (dir.), Mots. Les langages du politique, $n^{\circ} 112$, Discours présidentiels et de présidentielles, 2016

BALLET Marion, Émotions et élections. Les campagnes présidentielles françaises (1981-2012), INA Éditions, Paris, 2014

BARTHES Roland, Leçon, Seuil, Paris, (1977) 2015

BRAUD Philippe, L'Émotion en politique, Presses Universitaires de Sciences Po, Paris, 1996

CARLóN Mario, "Sur les pas de Verón... Une approche des nouvelles conditions de circulation du sens à l'ère contemporaine ", Traduction de Gustavo Gomez-Mejia \& Odile Cortinovis, Communication \& langages, $n^{\circ} 196$, Eliseo Verón. Vers une sémio-anthropologie, 2018, p.99-120

CHARAUDEAU Patrick, Le Discours politique. Les masques du pouvoir, Vuibert, Paris, 2005

CHARAUDEAU Patrick, "La pathémisation à la télévision comme stratégie d'authenticité ", Les Émotions dans les interactions, Presses universitaire de Lyon, 2000

DAYAN Daniel et KATZ Elihu, La Télévision cérémonielle. Anthropologie et histoire en direct, PUF, Paris, 1996

DEVARS Thierry, “'Ces mots qui résonnent': médiagénie du clash politique ", in AïM Olivier et al. (éds), Persistances benjaminiennes, $n^{\circ} 21$, Collection "Théorème", Presses Sorbonne Nouvelle, Paris, 2014, p.41-42

ELIAS Norbert, La Civilisation des mœurs, Calmann-Lévy, Paris, 1973

FAURE Alain \& NÉGRIER Emmanuel (dir.), La Politique à l'épreuve des émotions, Rennes, Presses universitaires de Rennes, coll. "Res Publica", 2017

FOESSEL Michqël (coord.), Esprit, Volume mars-avril, $n^{\circ}$ 3, Colères, 2016

GAXIE Daniel, La démocratie représentative, Montchrestien, Paris, 1996

HEINICH Nathalie, De Ia visibilité. Excellence et singularité en régime médiatique, Gallimard, "Bibliothèque des sciences humaines", Paris, 2012

LE BART Christian, "La représentation politique comme lien émotionnel", Nouvelles perspectives en sciences sociales, Volume $14, n^{\circ} 1$, sur le thème:
LE BART Christian, Les Émotions du pouvoir. Larmes, rires, colères des politiques, Armand Colin, Paris, 2018

LEFEBVRE Rémi, Leçons d'introduction à la science politique, Ellipses, Paris, 2010

LEROUX Pierre et RIUTORT Philippe, La politique sur un plateau. Ce que la télévision fait à la représentation, PUF, Paris, 2013

MARCUS Georges E., Le Citoyen sentimental: émotion et politique en démocratie, Les Presses de Sciences Po, Paris, 2008

MARION Philippe, “Narratologie médiatique et médiagénie des récits", Recherches en communication, $n^{\circ} 7, \mathrm{UCL}$, Louvain-la-Neuve, 1997

MERCIER Arnaud, PIGNARD-CHEYNEL Nathalie (dir.), \#info. Commenter et partager l'actualité sur Twitter et Facebook, Éditions de la Maison des sciences de l'homme, collection "Le (bien) commun ", Paris, 2018

RENAUT Olivier, "La colère du juste", Esprit, Volume mars-avril, $n^{\circ} 3,2016$, p. 135-145

SPINOZA Baruch, L'Éthique, Traduction par Charles Appuhn, Garnier Frères, (1677) 1913

TAVERNIER Aurélie, "Rhétoriques journalistiques de médiatisation ", Questions de communication, $\mathrm{n}^{\circ} 16,2009$, p.71-96

TRAIINI Christophe et BLONDIAUX Loïc (dir.), La démocratie des émotions. Dispositifs participatifs et gouvernabilité des affects, Presses de Sciences Po, Paris, 2018

TRAIINI Christophe (dir.), Émotions... Mobilisation!, Presses de Sciences Po, Paris, 2009

VERÓN Eliseo, "El living y sus dobles. Arquitecturas de la pantalla chica", El cuerpo de las imágenes (1984), Buenos Aires, Norma, 2001, p. 13-40 